\title{
A practical guide to event generation for prompt photon production with Sherpa
}

\author{
Frank Siegert \\ Institut für Kern- und Teilchenphysik, TU Dresden, D-01062 Dresden, Germany \\ E-mail: frank.siegert@cern.ch
}

\begin{abstract}
.
The production of prompt photons is one of the most relevant scattering processes studied at hadron-hadron colliders in recent years. This article will give an overview of the different approaches used to simulate prompt photon production in the Sherpa event generator framework. Special emphasis is placed on a complete simulation of this process including fragmentation configurations. As a novel application a merged simulation of $\gamma \gamma$ and $\gamma \gamma+$ jet production at NLO accuracy is presented and compared to measurements from the ATLAS experiment.
\end{abstract}

PACS numbers: 12.38.-t, 12.38.Bx, 12.38.Cy, 13.85.Hd, 14.70.Bh

\section{Introduction}

Prompt photon pairs have constituted one of the most relevant signatures in collider measurements in the recent years. Not only the discovery of the Higgs boson [1] was first announced in that channel, but also several further searches for unknown heavy particles decaying into photons have been performed by the LHC experiments [2] and tracked closely by the theory community.

Though clearly the spotlight is on resonant production, one crucial ingredient for such a physics programme to succeed has been the understanding of the backgrounds from continuum diphoton production. In the direct search for resonances this is often implemented by a fit to data from the invariant mass distribution using the sideband regions next to the region of interest, e.g. using various polynomial functions [2].

This is problematic as soon as one is looking for heavy resonances. Here, the fit of the functional parameters becomes unstable because the high-mass sideband region contains only a low number of events in data. Thus, it becomes crucial to have a theory-based prediction, which will constrain the template fit of the Standard Model background more strongly.

Despite its relevance, diphoton production has not been a very popular testbed for the development and application of new and precise event generation techniques up to now. This is partially due to its narrow scope of application as a background process 
in searches for new particles, but to a large extent has to be blamed on the intricate difficulties of prompt photon production.

For example, the simultaneous production of photons and partons in any higherorder calculation induces additional collinear divergences, which have to be regularised and/or factorised into a fragmentation component. Furthermore, the loop-induced gluon-gluon initial-state process, even though formally only relevant at higher orders, is enhanced by the high gluon density in the proton at LHC energies. Last, but not least, the calculation of amplitudes with many photons and partons is challenging, which is demonstrated by the slower progress in calculating NLO high-multiplicity amplitudes for $\gamma \gamma+$ jets [3] as compared to e.g. $V+$ jets [4].

Theoretical predictions for prompt photon production are usually based on fixedorder perturbation theory, assuming the application of suitable isolation criteria [5, 6] to remove infrared singularities. Additionally, a resummation of the large logarithms induced by QCD corrections can be described semi-analytically by photon fragmentation functions [7]. They are conceptually similar to a parton distribution function but describe the probability for the transition of a parton into a collinear photon with a given momentum fraction.

Perturbative calculations of higher-order QCD corrections to single photon and diphoton production were performed in the JETPHOX [8] and DIPHOX [9] programs at NLO, for the first time including also the full fragmentation contribution. Furthermore, in recent years also NNLO accurate results for diphoton production have appeared [10] and yield an improved agreement with experimental measurements [11, 12].

For usage in experimental measurements and direct comparisons to those, it is necessary to go beyond analytical calculations and include hadronisation and underlyingevent effects in the prediction. These are typically generated by parton shower event generators, which not only include a phenomenological modelling of hadronisation and multiple particle interactions but also add to the fixed-order calculation a resummation of large logarithms due to collinear parton emissions, thus providing an alternative to the usage of analytical fragmentation functions. Several modern Monte Carlo event generators [13, 14, 15, 16] are going beyond the simple parton-shower based approach and allow for a more precise simulation of prompt photon processes.

This article consists of two parts: Section 2 contains a pedagogical overview of the traditional and modern methods for the simulation of photon production in the hadron collider event generator Sherpa. In Section 3 the Sherpa framework is then used to introduce for the first time a merged simulation of $\gamma \gamma$ and $\gamma \gamma+$ jet at NLO QCD accuracy. After presenting the results obtained with this simulation the article will conclude and give an outlook towards further work.

\section{Overview of prompt photon event generation in Sherpa}

This section contains a short overview of how prompt photon production is simulated in full event generators like Sherpa. It starts from the basic parton shower picture, and 


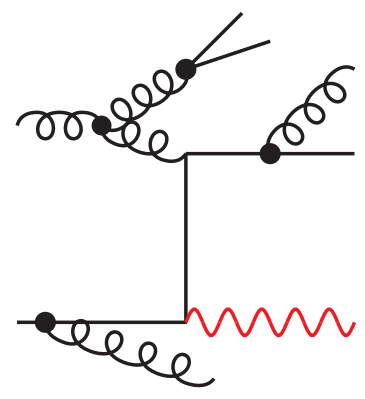

(a) Direct photons

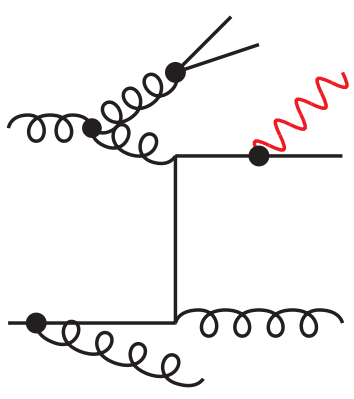

(b) Fragmentation photons

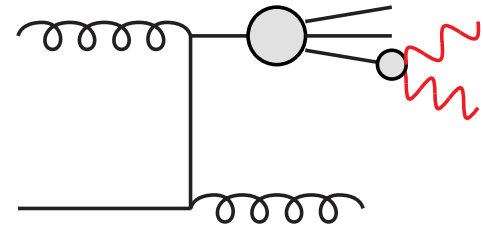

(c) Non-prompt photons

Figure 1: Photon production mechanisms in traditional parton shower programs. The black dots represent parton shower splittings, while the grey circles represent hadronisation and hadron decay processes.

then describes the more advanced approaches. While the description is mostly kept general, the details and examples will refer to the implementation in the Sherpa event generator [17].

\subsection{Basic parton shower}

In a traditional parton shower simulation, based on $2 \rightarrow 2$ matrix elements and a subsequent parton cascade, photons can be produced by three different mechanisms:

(i) Direct production

Matrix elements for $p p \rightarrow \gamma+X$ production are calculated at the leading order and the parton shower adds QCD emissions, cf. Fig. 1a.

This implies a strict hierarchy of scales in the shower evolution variable $t$, with QCD parton emissions $t_{\mathrm{QCD}}$ allowed only at a lower (softer) scale than the factorisation scale defined by the direct photon production process $t_{\mathrm{QED}}$.

(ii) Fragmentation production

Parton showers can be extended straightforwardly to include not only QCD splitting functions, but also their QED equivalent [18]. Thus, photons can be emitted from quark lines, resumming the corresponding logarithmic enhancement from collinear configurations.

This implies a combined shower evolution with a "competition" for the emission phase space between QED and QCD emissions. Since QCD and QED emissions do not interfere, the Sudakov form factor takes a factorised form [13]:

$$
\Delta\left(\mu_{0}^{2}, Q^{2}\right)=\Delta^{\mathrm{QCD}}\left(\mu_{0}^{2}, Q^{2}\right) \Delta^{\mathrm{QED}}\left(\mu_{0}^{2}, Q^{2}\right),
$$

where the QED part,

$$
\Delta^{\mathrm{QED}}\left(\mu_{0}^{2}, Q^{2}\right)=\exp \left\{-\int_{\mu_{0}^{2}}^{Q^{2}} \frac{\mathrm{d} t}{t} \int \mathrm{d} z \sum_{i} \frac{1}{2} \mathcal{K}_{i}^{\mathrm{QED}}(z, t)\right\},
$$



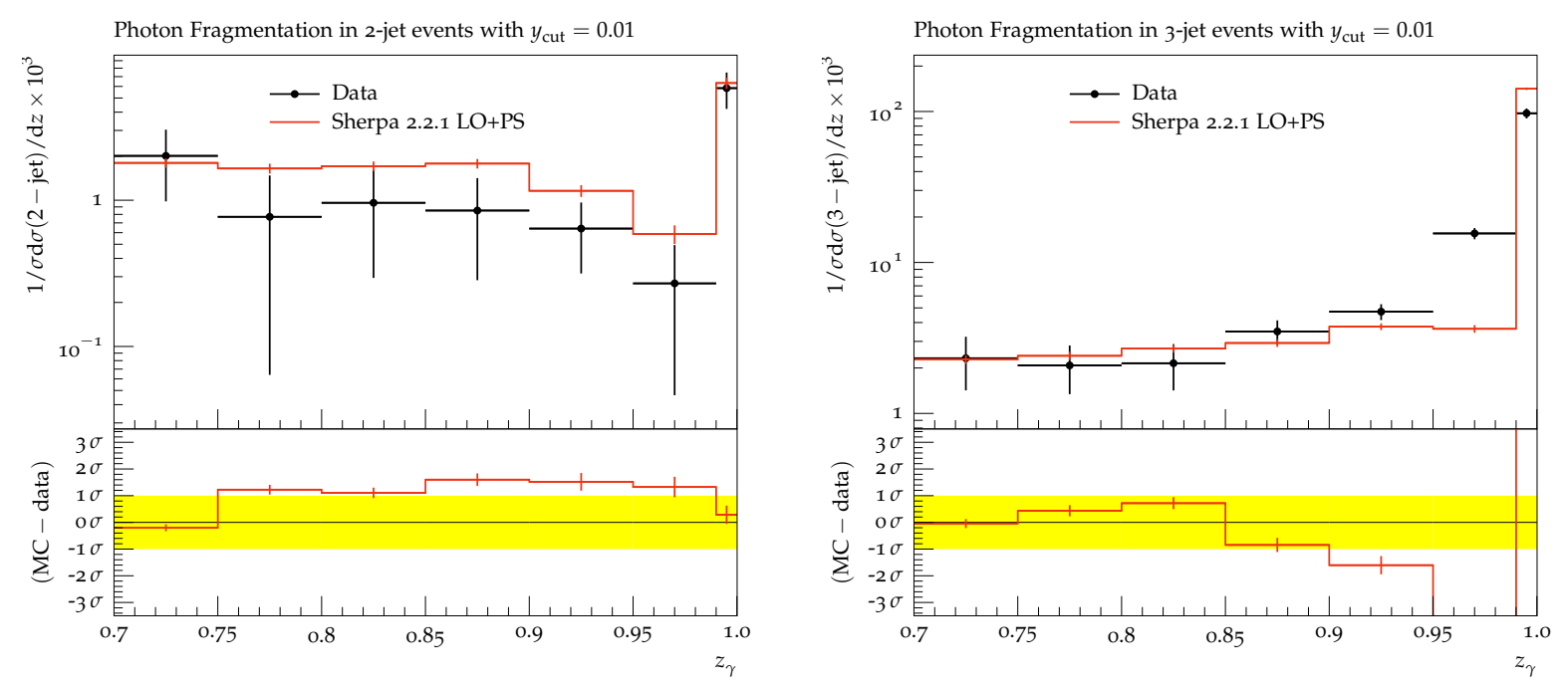

Figure 2: Fragmentation distributions measured by the ALEPH experiment in comparison with a QED shower prediction from Sherpa 2.2.1.

contains the QED splitting functions $\mathcal{K}_{i}^{\mathrm{QED}}(z, t)$, in direct analogy to the QCD part.

The production of photon pairs through this fragmentation mechanism requires the inclusion of matrix elements for $p p \rightarrow \gamma+$ jet (single fragmentation) and even $p p \rightarrow$ jet + jet (double fragmentation) in the simulation. A pictorial representation is shown in Fig. $1 \mathrm{~b}$,

Again, as this is a parton shower, one encounters a strict hierarchy of scales opposite to the case above, with the QED emissions $t_{\mathrm{QED}}$ being softer than the core parton production process $t_{\mathrm{QCD}}$.

(iii) Non-prompt production

Hadrons decaying into photons, like $\pi^{0} \rightarrow \gamma \gamma$, see Fig. 1c, represent a photon contribution that is always present, but can be disentangled from the prompt production above in a physical and to some extent even experimentally meaningful way, and shall thus not be discussed in the course of this work.

The fragmentation production in a parton shower can be probed very specifically by looking at photons in jets produced in electron-positron collisions. To demonstrate the QED shower implementation in Sherpa 2.2.1 [13], it is compared to the measurement of fragmentation distributions by the ALEPH experiment [19] in Fig. 2. Those are differential in the energy fraction the photon carries within the jet, $z_{\gamma}$, and shown for 2- and 3-jet events. While the experimental uncertainties are very large, and the agreement is not perfect in all bins, the general features of the fragmentation distribution are described fairly well already by this simple parton shower prediction.

It should be noted, that direct and fragmentation production cannot be separated in a physical way. The division into these two parts is merely a technical detail of the calculation or simulation, and they cannot be separately measured. 

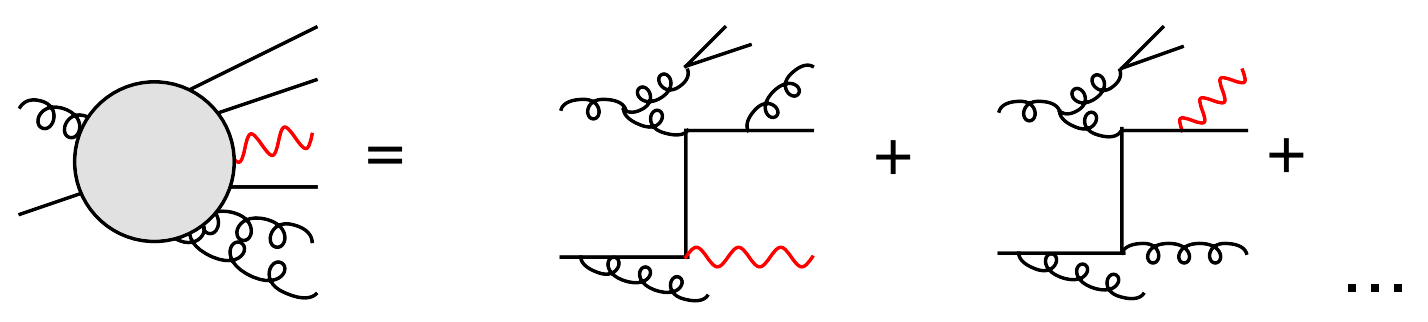

Figure 3: Example for multi-jet process within QCD-merged setup, demonstrating the inclusion of the contributions from Figs. $1 \mathrm{a}$ and $1 \mathrm{~b}$.

There are two significant drawbacks of the pure parton shower approach.

From a purely practical point of view, the prediction of the fragmentation component can be tedious, as the combination of a low probability for the emission of a hard and isolated photon with the high cross section of the underlying $2 \rightarrow 2$ processes requires the generation of incredibly large event samples. This can be mitigated to some extent by using shower programs where it is possible to enhance the QED splitting processes using appropriately weighted events [13].

But more importantly, the accuracy of the simulation is at best leading order (for the direct component) or even takes into account only the leading logarithms through the parton shower resummation (for the fragmentation component).

Nevertheless, these methods allow to reach a fair agreement with prompt photon production data from experiments [11, 12].

\subsection{Tree-level QCD merging}

Parton-shower simulations can be improved by the inclusion of tree-level QCD multi-jet matrix elements in a consistent merging scheme [20, 21]. Such simulations have become the workhorses of LHC experiments for the simulation of final states including multiple jets and can be applied to prompt photon final states as well.

The main idea is the introduction of a separation criterion $Q_{i j}$ for partons $i, j$ which is then used to slice the phase space for parton emissions from a given core process $p p \rightarrow X$ into two domains through a merging cut $Q_{\text {cut }}$ :

ME domain $Q_{i j}>Q_{\text {cut }}$

This region is populated by the tree-level matrix element including an additional parton, $p p \rightarrow X+$ jet.

PS domain $Q_{i j}<Q_{\text {cut }}$

This region is filled using the core matrix element $p p \rightarrow X$ and performing a parton shower emission.

It becomes particularly interesting when this is applied to prompt photon production, depicted in Fig. 3. There is no longer the immediate correspondence of "direct $\equiv$ ME" and "fragmentation $\equiv$ PS" production. Instead, the multi-jet matrix 
elements will now also contribute to the phase space which was previously covered by the QED parton shower.

Trying to relate these to each other, it is instructive to consider the scale hierarchies mentioned in Sec. 2.1. For example, considering the first emission, the corresponding matrix element covers the phase space including both hierarchies:

$$
\begin{array}{ccc}
Q_{i j}>Q_{\text {cut }} \cup & t_{\mathrm{QCD}}<t_{\mathrm{QED}} \\
Q_{i j}>Q_{\text {cut }} & \cup \quad t_{\mathrm{QCD}}>t_{\mathrm{QED}} .
\end{array}
$$

The QED shower is disabled in such a scheme, and all photon emissions will be generated directly with matrix-element accuracy. However, the replacement of the QED shower by higher-order tree-level matrix elements also comes with disadvantages.

The inclusion of high multiplicity matrix elements in the QCD merging approach makes it necessary to manually impose a regularisation of the infrared singularities of the photon in multi-parton configurations. To this end, phase space cuts are applied, which mimick experimental requirements for the transverse momentum of the photon and its isolation with respect to hadronic activity. The event generation is thus not fully inclusive anymore with respect to the photon, and care has to be taken to not introduce a bias for example for events after detector simulation.

Depending on how inclusive these photon cuts are chosen, it can also become problematic, that the resummation of collinear QED emission terms is not included anymore in the QCD merged sample. But in practice, this will be mitigated by the typical photon energy and isolation cuts in any prompt photon analysis.

\subsection{Tree-level $Q C D \otimes Q E D$ merging}

A natural extension of the QCD ME+PS merging approach to QED emissions leads to a solution of both problems just mentioned. In a combined QCD $\otimes$ QED merging, one incorporates QED emissions from both the parton shower and the hard scattering matrix elements. To avoid the resulting double-counting, the parton separation criterion $Q_{i j}$ is extended also to photons, effectively imposing a democratic merging of photons and partons.

To demonstrate first the QED merging component alone, lepton pair production at the $13 \mathrm{TeV}$ LHC is chosen as a testbed. The benchmark observable in Fig. 4 is the $Z$-lineshape which is sensitive to QED emissions, in particular when built from "bare" leptons (left) as opposed to "dressed" leptons (right), which are built by including photon radiation in a cone of $R=0.1$ around the lepton. The QED merging is here tested using an extremely low merging scale, $Q_{\text {cut }}=0.1 \mathrm{GeV}$, to have any effect on this observable at all. Matrix elements for the $p p \rightarrow \ell \ell$ production process with up to two additional photons are included in the merged sample.

The results from this exercise demonstrate that such a merging approach could solve both disadvantages mentioned in the last section: 

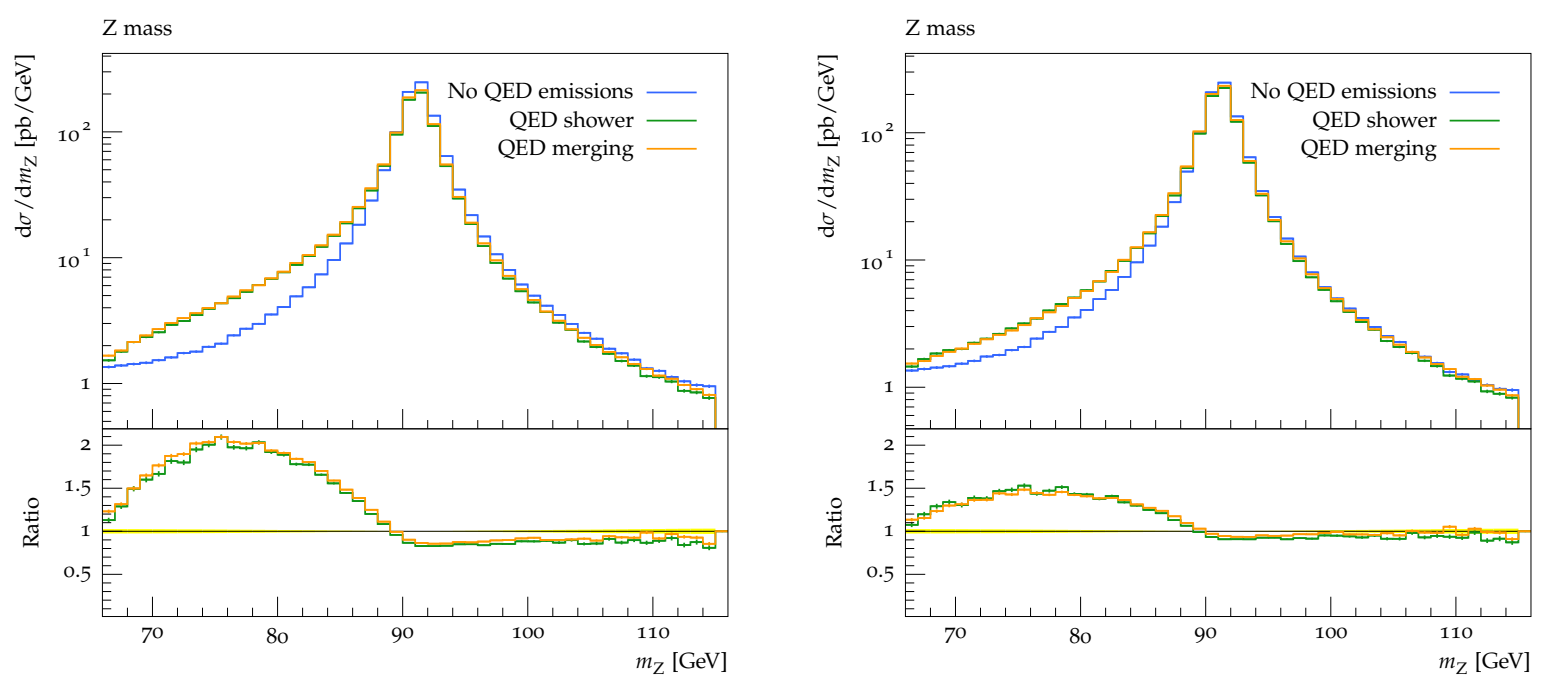

Figure 4: QED merging validation in the $Z$-lineshape in lepton-pair production at the $13 \mathrm{TeV}$ LHC, using bare leptons (left) and dressed leptons (right).

The merged prediction reproduces the shower prediction for the lineshape, i.e. the resummation of QED emissions is implemented correctly in this approach, solving the first problem mentioned earlier.

Furthermore, also the inclusiveness of the event sample with respect to any potential photon cuts is restored: the cuts applied to the higher-multiplicity matrix elements now become merging scales, which only govern the transition to the parton shower emissions, that fill the phase space.

The combined QCD $\otimes$ QED merging can now be implemented in a straightforward way and can thus be applied to prompt photon production by combining matrix elements for multi-parton and multi-photon production completely democratically.

At this point the reader may argue that one is impacted again by the inefficiencies of the QED parton shower discussed in Sec. 2.1. In other words, the relative cross section of the tree-level matrix elements containing parton production will be orders of magnitude larger than the matrix elements with outgoing photons - but both appear within the same merged setup. While this large cross section difference is, of course, compensated by the low probability to produce a hard isolated photon in the QED shower, it remains impractical to generate such a sample for hard isolated photons.

This issue can be mitigated by choosing the merging cut for photon emissions smartly, such that QED shower emissions are not relevant for the analysis region. One is at liberty to choose a different value of $Q_{\text {cut }}$ for QED emissions compared to QCD emissions. This is reasonable because it reflects the different behaviour of both the theory in the infrared limit and the experimental resolution. Since the merging criterion is a technical parameter which merely has to reproduce the proper singular limits, one can even choose a different functional form of $Q_{i j}$ for the case where $i$ or $j$ is a photon. In effect, one can adapt the merging cut to reflect the energy and isolation cuts of hard isolated photons in the analysis. For example, in the common case where a minimal 
transverse momentum and a spatial separation $\Delta R_{i j}^{2}=\Delta \eta_{i j}^{2}+\Delta \phi_{i j}^{2} \geq D$ are required one could choose the equivalent of the longitudinally invariant $k_{t^{-}}$algorithm [22, 13]:

$$
Q_{i j}^{2}=\min \left\{p_{\perp, i}^{2}, p_{\perp, j}^{2}\right\} \frac{\Delta R_{i j}^{2}}{D^{2}} \text { and } Q_{i b}^{2}=p_{\perp, i}^{2},
$$

where $i$ is always a photon, $j$ is a charged final-state particle, and $b$ represents a charged incoming particle.

Going even further, with an appropriately chosen merging cut, the contributions due to the QED shower can be ignored in the final event generation sample, and be generated merely as a separate low-statistics sample to cross-check that they are indeed not contributing to the region of interest.

Instead of pursuing this route further, let us now turn to a new approach to generate prompt photon production at higher accuracies.

\section{Merged diphoton production at NLO QCD accuracy}

The existing methods for hadron-level event generation described earlier all have one significant drawback compared to state-of-the-art fixed-order calculations: they are only accurate to leading order in QCD perturbation theory.

Extending NLO accuracy to the full QCD $\otimes$ QED merging setup as described in Sec. 2.3 would necessitate the inclusion of higher order corrections not only in QCD but also in QED. Some of the building blocks necessary for such a combined evolution at NLO accuracy are already available, such as NLO QED matrix elements including dedicated subtraction schemes for QED emissions [23], and leading-order QED splitting functions as implemented in the parton showers described in Sec. 2.1. But several crucial ingredients for a NLO QCD $\otimes$ QED merging are missing, such as the matching of QED real emission matrix elements with the QED parton shower, as well as the implementation of the subtracted truncated shower veto in the QED part of the MEPS@NLO merging [24].

One could even go one step further, beyond the factorised combination of QCD and QED corrections, and aim for a simulation with the full combined $\mathcal{O}\left(\alpha_{s} \alpha\right)$ corrections. To that end, the parton shower would have to be extended to be fit for NLO splitting functions and contain kernels up to $\mathcal{O}\left(\alpha_{s} \alpha\right)[25]$.

Here, an intermediate step is presented, allowing to achieve NLO QCD accuracy as an extension of the tree-level QCD merging approach described above. While there has been previous work to match NLO matrix elements for the inclusive diphoton production process with a parton shower [15, 16], this article goes one step further and includes NLO accuracy for both $p p \rightarrow \gamma \gamma$ and $p p \rightarrow \gamma \gamma+$ jet production in a merged simulation. This allows to benefit from the features introduced in Sec. 2.2, in particular the generation of the fragmentation component with the help of higher-multiplicity matrix elements instead of the QED shower. 


\subsection{Programs and setup}

The studies shown in the following are done using the Sherpa event generator framework [17], including a parton shower based on Catani-Seymour subtraction terms [26], tree-level matrix elements from the Amegic [27] and Comix [28] generators and one-loop matrix elements from OpenLoops [29]. The default Sherpa 2.2 tuning is used together with the NNPDF 3.0 NNLO PDF set [30].

The matching of NLO QCD matrix elements with the parton shower is implemented using a variant [31] of the original MC@NLO method [32].

$\mathrm{NLO}+\mathrm{PS}$ matched simulations for $p p \rightarrow \gamma \gamma$ and $p p \rightarrow \gamma \gamma+$ jet production are then merged into an inclusive sample using the MEPS@NLO approach [24]. Additionally, matrix elements with up to three partons in the final state are included at LO accuracy in the approach of [33].

The QCD factorisation and renormalisation scales for the diphoton core process are set to the invariant mass of the photon pair. The QED coupling constant is set to $\alpha=1 / 137.036$. Additional QCD couplings in the higher-multiplicity matrix elements are evaluated at their reconstructed branching scale in the formalism of [21].

The matrix elements are generated with cuts on the transverse momentum of the leading (subleading) photon, $p_{\perp}^{\gamma}>21(18) \mathrm{GeV}$. Photons are required to be central within $\left|y_{\gamma}\right|<2.7$ and isolated at the parton level according to a smooth cone isolation [6] with parameters $\delta=0.1, n=2$ and $\epsilon=0.1$. Additionally, a separation cut between both photons of $\Delta R\left(\gamma_{1}, \gamma_{2}\right)>0.2$ is applied.

\subsection{Dynamical merging scale}

As NLO accurate QCD $\otimes$ QED merging is not available in current event generators, one has to resort to the QCD merging approach as described in Sec. 2.2, but now at the NLO accuracy level. To deal with the limitations of the QCD merging approach, the following idea is implemented:

The main problem, namely the limited inclusiveness of the "fragmentation" component, concerns the inverse hierarchy configurations. While the ME domain part, Eq. (4), is included, the complementary contribution stemming from a QCD parton shower emission,

$$
Q_{i j}<Q_{\text {cut }} \quad \cup \quad t_{\mathrm{QCD}}>t_{\mathrm{QED}}
$$

would be necessary to generate the full "fragmentation" component. Since the parton shower cannot generate emissions with this inverse hierarchy, this contribution remains uncovered.

At first sight, this does not appear to be a significant issue, since the phase space described in Eq. (6) requires an emission which is still in the PS domain but harder than the photon production scale, thus naively making this contribution negligible. But in the case of prompt photon production there is no hard (mass) scale in the process 
which would automatically set a lower boundary for the shower starting scale. Hence this phase space region can become significant.

To solve this problem, one can make use of an approach which has been pioneered in a similar situation with low factorisation scales, namely for QCD merging in DIS [34].

The basic idea is to introduce a dynamical merging cut $Q_{\text {cut }}$ such that it is smaller than the shower starting scale $t_{\mathrm{QED}}$ at each phase space point. The shower starting scale is usually given by the factorisation scale of the prompt photon production process, i.e. something like $\mu_{F}=p_{\perp}^{\gamma}$ for single photon production or $\mu_{F}=m_{\gamma \gamma}$ for photon pair production. The dynamical merging cut can then be built from a fixed value $\bar{Q}_{\text {cut }}$ and the factorisation scale $\mu_{F}$ as:

$$
Q_{\text {cut }}=\left[\frac{1}{\bar{Q}_{\mathrm{cut}}^{2}}+\frac{1}{S^{2} \mu_{F}^{2}}\right]^{-1 / 2} .
$$

This particular form has the advantage, that it provides a smooth interpolation between the fixed value and the factorisation scale, but is dominated by the smaller of the two. The safety factor $S \lesssim 1$ can be chosen according to the parton shower model. The fixed component $\bar{Q}_{\text {cut }}$ is present in this dynamical scale to ensure that matrix element accuracy is used for hard emissions in phase space regions with high $\mu_{F}$.

\subsection{Results}

The approach described above is validated with a comparison to measurements from the ATLAS experiment [11] through their implementation in Rivet [35].

Events of proton-proton collisions at $\sqrt{s}=7 \mathrm{TeV}$ are selected to contain two isolated photons with transverse energies above $25 \mathrm{GeV}$ and $22 \mathrm{GeV}$, both within $\left|y_{\gamma}\right|<1.37$ or $1.52<\left|y_{\gamma}\right|<2.37$ and with $\Delta R(\gamma, \gamma)>0.4$. To define isolated photons, the hadronic energy within a cone of $\Delta R<0.4$ but excluding the inner core of $|\Delta \eta|<0.0625$ and $|\Delta \Phi|<\frac{7 \pi}{256}$ is required to be $E_{\text {cone }}<4 \mathrm{GeV}$. The energy is corrected taking into account the median transverse energy density in this region on an event-by-event basis to limit the sensitivity to multiple parton and proton interactions.

Predictions are obtained both for the tree-level as well as NLO QCD merging approach using the programs and setup described in Sec. 3.1. The dynamical merging scale introduced in Sec. 3.2 is used in both cases with $\bar{Q}_{\text {cut }}=10 \mathrm{GeV}$. In addition to the central prediction an uncertainty band is estimated from a 7-point independent scale variation of the factorisation and renormalisation scales by a factor of two up and down, excluding only opposite variations. Furthermore central predictions from switching the PDF set in the matrix element to CT14nnlo [36] and MMHT2014nnlo [37] are displayed. These variations are calculated using on-the-fly event weights in the implementation of [38].

Comparing the LO and NLO predictions for the total fiducial cross section in Table 1 one can draw two conclusions: The MEPS@NLO prediction agrees much better with the measured value than the MEPS@LO prediction. Both are compatible within uncertainties, but the size of the uncertainties in the former is reduced significantly. 


\begin{tabular}{lll}
$\sigma_{\text {MEPS@LO }}[\mathrm{pb}]$ & $\sigma_{\text {MEPS@NLO }}[\mathrm{pb}]$ & $\sigma_{\text {ATLAS }}[\mathrm{pb}]$ \\
\hline $33.9_{-5.9(18 \%)}^{+9.6(28 \%)}$ & $44.8_{-6.5(15 \%)}^{+6.7(15 \%)}$ & $44.0_{-4.2(10 \%)}^{+3.2(7 \%)}$
\end{tabular}

Table 1: Total fiducial cross sections and systematic uncertainties from MEPS@LO and MEPS@NLO predictions compared to the ATLAS measurement.

A more detailed picture emerges from the study of the differential distributions displayed in Figs. 5 and 6 .

The LO predictions show strong shape deviations from data in the region of low transverse momentum of the photon pair, yielding a deficit of up to $40 \%$. This is strongly improved in the NLO predictions, which agree well with data in this region which is significantly affected by fragmentation photons. Overall, the shape of the $p_{\perp \gamma \gamma}$ spectrum is described very well throughout the whole range in the NLO prediction, including the resummation region $p_{\perp \gamma \gamma} \rightarrow 0$, the hard region $p_{\perp \gamma \gamma}>100 \mathrm{GeV}$, and the intermediate region including the well-known shoulder.

The azimuthal decorrelation of the two photons reveals larger shape differences between the two predictions in the region $\frac{\pi}{2}<\Delta \phi<\pi$, with the NLO prediction providing a shape more compatible with what is found in data.

The invariant mass distribution of the photon pair and the polar angle of the harder photon in the Collins-Soper frame exhibit only minor differences between the shapes of the two predictions. The suppressed region below the peak in the $m_{\gamma \gamma}$ spectrum and the region of $\left|\cos \theta^{*}\right| \rightarrow 1$ are slightly underestimated in both cases.

Let us now turn to a discussion of the systematic uncertainties. While they have been significantly reduced when considering the total cross section, the uncertainties in specific regions of phase space still seem fairly large when going from LO to NLO.

To understand this feature, it is instructive to first consider the multi-jet process composition demonstrated by the dashed lines in the ratio plots. Some regions like $p_{\perp \gamma \gamma}>100 \mathrm{GeV}$ and $\Delta \phi_{\gamma \gamma} \rightarrow 0$ are dominated by hard multi-jet emissions, i.e. the blue and green dashed lines corresponding to 2 and 3 jets at the parton level, respectively.

These processes are simulated here only at leading order accuracy. Thus they will lead to larger systematic uncertainties as seen in the increased size of the scale variation band in these regions. This effect is amplified as one is using a dynamical merging scale which can extend to very small values. For a small value of the merging scale the cross section of the LO matrix elements for $p p \rightarrow \gamma \gamma+2,3$ jets is increased relative to the NLO $p p \rightarrow \gamma \gamma+0,1$ jets matrix elements. On the other hand, if the merging scale would be chosen less inclusive, parts of the fragmentation component would be missing in the sample. Furthermore, in that case the additional emissions would be modelled by the parton shower, which would also induce larger uncertainties that are not included in the bands displayed here.

On the other hand, in regions dominated by the NLO-accurate process simulation one finds uncertainties comparable in size to the current experimental uncertainties, thus 

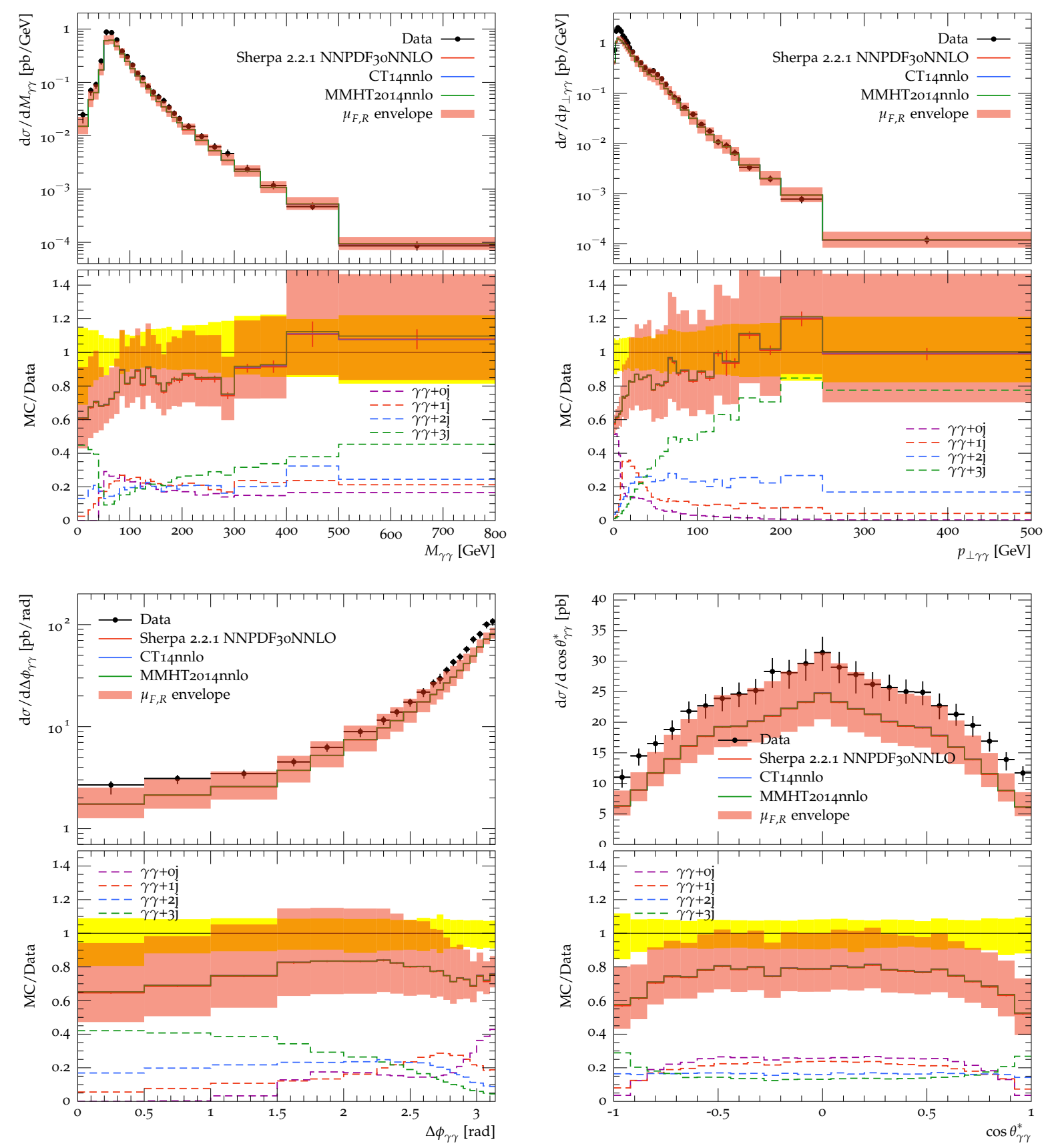

Figure 5: MEPS@LO predictions for diphoton production at the $7 \mathrm{TeV}$ LHC for the invariant mass (top left), transverse momentum (top right) and azimuthal separation (bottom left) of the photon pair and the polar angle of the harder photon in the CollinsSoper frame (bottom right). Data from the ATLAS experiment [11] is represented by the black markers and the yellow uncertainty band. The solid red line represents the central prediction and the red markers (band) show the corresponding statistical (systematic) uncertainties. The solid blue and green line show predictions using different PDF sets as described in the main text. The dashed coloured lines in the ratio plot demonstrate the composition of the central prediction from different jet multiplicities at the parton level. 

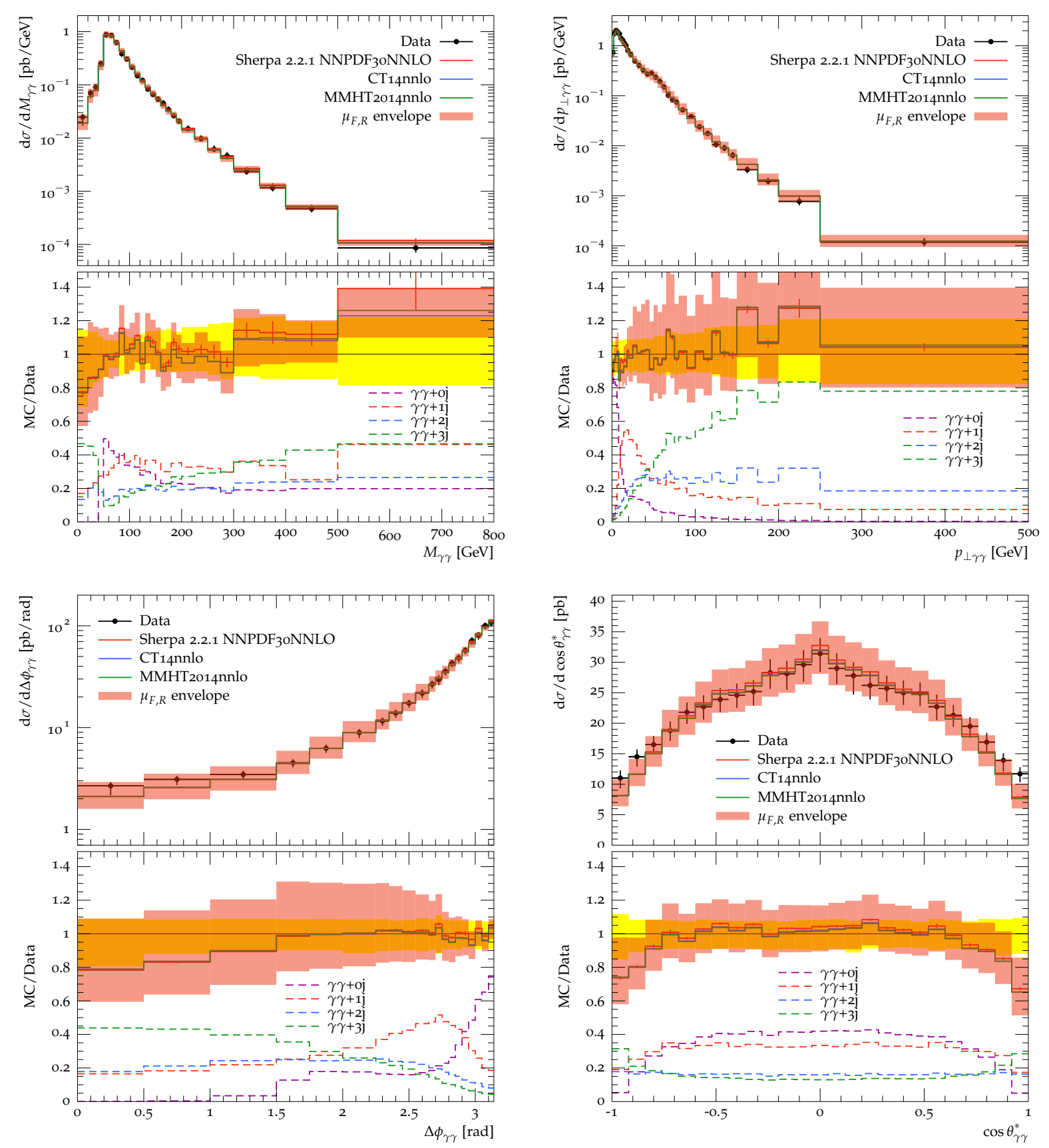

Figure 6: MEPS@NLO predictions for diphoton production at the $7 \mathrm{TeV}$ LHC for the invariant mass (top left), transverse momentum (top right) and azimuthal separation (bottom left) of the photon pair and the polar angle of the harder photon in the CollinsSoper frame (bottom right). Data from the ATLAS experiment [11] is represented by the black markers and the yellow uncertainty band. The solid red line represents the central prediction and the red markers (band) show the corresponding statistical (systematic) uncertainties. The solid blue and green line show predictions using different PDF sets as described in the main text. The dashed coloured lines in the ratio plot demonstrate the composition of the central prediction from different jet multiplicities at the parton level. 
demonstrating the improvements due to the application of the MEPS@NLO merging approach.

\section{Conclusions}

A pedagogical overview of prompt photon production in the parton shower event generator Sherpa was given. Modern generators like Sherpa improve the precision of these predictions by including exact higher-order corrections in QCD perturbation theory. A particular difficulty in the context of prompt photon production lies in the inclusive description of the fragmentation component. The extent to which this is possible in different approaches has been discussed.

New predictions for the production of a prompt photon pair with one additional jet at NLO QCD accuracy and matched and merged with lower and higher multi-jet configurations were presented for the first time. Good agreement with measurements from the ATLAS experiment was found and the theoretical uncertainties have been studied.

The assessment of these uncertainties naturally leads to an outlook to future work. To improve predictions in the regions dominated by multi-jet production it is desirable to include NLO accurate matrix elements also for the higher multiplicities, as far as computationally feasible. The still sizable global uncertainty can be reduced only if a new scheme is devised that allows the inclusion of NNLO-accurate calculations for the inclusive process in such a merged sample. Furthermore, with additional work on the logarithmic accuracy of the parton shower one would reduce a source of uncertainties which have not been considered in this work and which might still play a large role in some regions of the observables shown.

\section{Acknowledgements}

We are grateful to Stefan Höche and Steffen Schumann for many fruitful discussions on the subject and manuscript. This research was supported by the German Research Foundation (DFG) under grant No. SI 2009/1-1. We thank the Center for Information Services and High Performance Computing (ZIH) at TU Dresden for generous allocations of computing time.

\section{References}

[1] G. Aad et al., The ATLAS collaboration, Observation of a new particle in the search for the Standard Model Higgs boson with the ATLAS detector at the LHC, Phys.Lett. B716 (2012), 1-29, arXiv:1207.7214 [hep-ex]]; S. Chatrchyan et al., The CMS collaboration, Observation of a new boson at a mass of $125 \mathrm{GeV}$ with the CMS experiment at the LHC, Phys.Lett. B716 (2012), 30-61, arXiv: 1207.7235 [hep-ex]].

[2] ATLAS collaboration, Search for scalar diphoton resonances with $15.4 \mathrm{fb}^{-1}$ of data collected at $\sqrt{s}=13 \mathrm{TeV}$ in 2015 and 2016 with the ATLAS detector, Tech. Report ATLAS-CONF-2016059. CERN, Geneva, Aug 2016; V. Khachatryan et al., The CMS collaboration, Search for 
high-mass diphoton resonances in proton-proton collisions at $13 \mathrm{TeV}$ and combination with 8 TeV search, arXiv: 1609.02507 [hep-ex].

[3] S. Badger, A. Guffanti and V. Yundin, Next-to-leading order QCD corrections to di-photon production in association with up to three jets at the Large Hadron Collider, JHEP 1403 (2014), 122, arXiv: 1312.5927 [hep-ph]].

[4] Z. Bern, L. Dixon, F. Febres Cordero, S. Höche, H. Ita, D. A. Kosower, D. Maître and K. J. Ozeren, Next-to-Leading Order W+5-Jet Production at the LHC, Phys.Rev. D88 (2013), 014025, arXiv: 1304.1253 [hep-ph]].

[5] E. W. N. Glover and A. G. Morgan, Measuring the photon fragmentation function at LEP, Z. Phys. C62 (1994), 311-322, S. Catani, M. Fontannaz, J.-P. Guillet and E. Pilon, Isolating Prompt Photons with Narrow Cones, JHEP 09 (2013), 007, arXiv:1306.6498 [hep-ph]].

[6] S. Frixione, Isolated photons in perturbative QCD, Phys. Lett. B429 (1998), 369-374. hep-ph/9801442.

[7] C. H. Llewellyn Smith, QCD predictions for processes involving real photons, Phys. Lett. B79 (1978), 83.

[8] S. Catani, M. Fontannaz, J.-P. Guillet and E. Pilon, Cross section of isolated prompt photons in hadron-hadron collisions, JHEP 05 (2002), 028, hep-ph/0204023; P. Aurenche, M. Fontannaz, J.-P. Guillet, E. Pilon and M. Werlen, Recent critical study of photon production in hadronic collisions, Phys. Rev. D73 (2006), 094007, hep-ph/0602133; Z. Belghobsi et al., Photonjet correlations and constraints on fragmentation functions, Phys. Rev. D79 (2009), 114024. arXiv:0903.4834 [hep-ph]].

[9] T. Binoth, J.-P. Guillet, E. Pilon and M. Werlen, A full next-to-leading order study of direct photon pair production in hadronic collisions, Eur. Phys. J. C16 (2000), 311-330, hep-ph/9911340; T. Binoth, J.-P. Guillet, E. Pilon and M. Werlen, Beyond leading order effects in photon pair production at the Fermilab Tevatron, Phys. Rev. D63 (2001), 114016, hep-ph/0012191].

[10] S. Catani, L. Cieri, D. de Florian, G. Ferrera and M. Grazzini, Diphoton production at hadron colliders: a fully- differential QCD calculation at NNLO, Phys.Rev.Lett. 108 (2011), 072001, arXiv:1110.2375 [hep-ph]]; J. M. Campbell, R. K. Ellis, Y. Li and C. Williams, Predictions for diphoton production at the LHC through NNLO in QCD, JHEP 07 (2016), 148, arXiv: 1603.02663 [hep-ph]].

[11] G. Aad et al., The ATLAS collaboration, Measurement of isolated-photon pair production in pp collisions at $\sqrt{s}=7 \mathrm{TeV}$ with the ATLAS detector, JHEP 01 (2013), 086, arXiv:1211.1913 [hep-ex]].

[12] T. Aaltonen et al., The CDF collaboration, Measurement of the Cross Section for Prompt Isolated Diphoton Production Using the Full CDF Run II Data Sample, Phys. Rev. Lett. 110 (2013), no. 10, 101801, [arXiv:1212.4204 [hep-ex]]; V. M. Abazov et al., The D0 collaboration, Measurement of the differential cross sections for isolated direct photon pair production in $p \bar{p}$ collisions at $\sqrt{s}=1.96 \mathrm{TeV}$, Phys. Lett. B725 (2013), 6-14, arXiv:1301.4536 [hep-ex]]; S. Chatrchyan et al., The CMS collaboration, Measurement of differential cross sections for the production of a pair of isolated photons in pp collisions at $\sqrt{s}=7 \mathrm{TeV}$, Eur. Phys. J. C74 (2014), no. 11, 3129, arXiv:1405.7225 [hep-ex]].

[13] S. Höche, S. Schumann and F. Siegert, Hard photon production and matrix-element parton-shower merging, Phys. Rev. D81 (2010), 034026, arXiv:0912.3501 [hep-ph]].

[14] S. Odaka and Y. Kurihara, Consistent simulation of non-resonant diphoton production in hadron collisions including associated jet production up to two jets, arXiv:1607.00204 [hep-ph].

[15] L. D'Errico and P. Richardson, Next-to-Leading-Order Monte Carlo Simulation of Diphoton Production in Hadronic Collisions, JHEP 02 (2012), 130, arXiv:1106.3939 [hep-ph]].

[16] T. Ježo, M. Klasen and F. König, Prompt photon production and photon-hadron jet correlations with POWHEG, arXiv: 1610.02275 [hep-ph].

[17] T. Gleisberg, S. Höche, F. Krauss, M. Schönherr, S. Schumann, F. Siegert and J. Winter, Event generation with Sherpa1.1, JHEP 02 (2009), 007, arXiv:0811.4622 [hep-ph]]. 
[18] M. H. Seymour, Photon radiation in final state parton showering, Z. Phys. C56 (1992), 161-170. M. H. Seymour, Soft isolated photon production as a probe of the parton shower mechanism, Z. Phys. C64 (1994), 445-452.

[19] D. Buskulic et al., The ALEPH collaboration, First measurement of the quark-to-photon fragmentation function, Z. Phys. C69 (1996), 365-378.

[20] S. Catani, F. Krauss, R. Kuhn and B. R. Webber, QCD matrix elements + parton showers, JHEP 11 (2001), 063, hep-ph/0109231; L. Lönnblad, Correcting the colour-dipole cascade model with fixed order matrix elements, JHEP 05 (2002), 046, hep-ph/0112284]; M. L. Mangano, M. Moretti and R. Pittau, Multijet matrix elements and shower evolution in hadronic collisions: $W b \bar{b}+n$-jets as a case study, Nucl. Phys. B632 (2002), 343-362, hep-ph/0108069; F. Krauss, Matrix elements and parton showers in hadronic interactions, JHEP 08 (2002), 015. hep-ph/0205283; K. Hamilton, P. Richardson and J. Tully, A modified CKKW matrix element merging approach to angular-ordered parton showers, JHEP 11 (2009), 038, arXiv:0905.3072 [hep-ph]]; L. Lönnblad and S. Prestel, Matching Tree-Level Matrix Elements with Interleaved Showers, JHEP 03 (2012), 019, arXiv:1109.4829 [hep-ph]].

[21] S. Höche, F. Krauss, S. Schumann and F. Siegert, QCD matrix elements and truncated showers, JHEP 05 (2009), 053, arXiv:0903.1219 [hep-ph]].

[22] S. Catani, Y. L. Dokshitzer and B. R. Webber, The $k_{\perp}$ clustering algorithm for jets in deep inelastic scattering and hadron collisions, Phys. Lett. B285 (1992), 291-299; S. Catani, Y. L. Dokshitzer, M. H. Seymour and B. R. Webber, Longitudinally-invariant $k_{\perp-\text {-clustering algorithms }}$ for hadron-hadron collisions, Nucl. Phys. B406 (1993), 187-224.

[23] S. Dittmaier, A general approach to photon radiation off fermions, Nucl. Phys. B565 (2000), 69-122, hep-ph/9904440; S. Dittmaier, A. Kabelschacht and T. Kasprzik, Polarized QED splittings of massive fermions and dipole subtraction for non-collinear-safe observables, Nucl. Phys. B800 (2008), 146-189, [arXiv:0802.1405 [hep-ph]].

[24] S. Höche, F. Krauss, M. Schönherr and F. Siegert, QCD matrix elements + parton showers: The NLO case, JHEP 04 (2013), 027, arXiv:1207.5030 [hep-ph]].

[25] D. de Florian, G. F. R. Sborlini and G. Rodrigo, QED corrections to the Altarelli-Parisi splitting functions, Eur. Phys. J. C76 (2016), no. 5, 282, arXiv:1512.00612 [hep-ph]].

[26] S. Schumann and F. Krauss, A parton shower algorithm based on Catani-Seymour dipole factorisation, JHEP 03 (2008), 038, |arXiv:0709.1027 [hep-ph]].

[27] F. Krauss, R. Kuhn and G. Soff, AMEGIC++ 1.0: A Matrix Element Generator In $C++$, JHEP 02 (2002), 044, hep-ph/0109036.

[28] T. Gleisberg and S. Höche, Comix, a new matrix element generator, JHEP 12 (2008), 039, arXiv:0808.3674 [hep-ph]].

[29] F. Cascioli, P. Maierhöfer and S. Pozzorini, Scattering Amplitudes with Open Loops, Phys.Rev.Lett. 108 (2012), 111601, arXiv:1111.5206 [hep-ph]].

[30] R. D. Ball et al., The NNPDF collaboration, Parton distributions for the LHC Run II, JHEP 04 (2015), 040, arXiv:1410.8849 [hep-ph]].

[31] S. Höche, F. Krauss, M. Schönherr and F. Siegert, A critical appraisal of NLO+PS matching methods, JHEP 09 (2012), 049, arXiv:1111.1220 [hep-ph]].

[32] S. Frixione and B. R. Webber, Matching NLO QCD computations and parton shower simulations, JHEP 06 (2002), 029 , hep-ph/0204244.

[33] S. Höche, F. Krauss, M. Schönherr and F. Siegert, NLO matrix elements and truncated showers, JHEP 08 (2011), 123, arXiv:1009.1127 [hep-ph]].

[34] T. Carli, T. Gehrmann and S. Höche, Hadronic final states in deep-inelastic scattering with Sherpa, Eur. Phys. J. C67 (2010), 73, arXiv:0912.3715 [hep-ph]].

[35] A. Buckley, J. Butterworth, L. Lönnblad, D. Grellscheid, H. Hoeth et al., Rivet user manual, Comput.Phys.Commun. 184 (2013), 2803-2819, arXiv:1003.0694 [hep-ph]].

[36] S. Dulat, T.-J. Hou, J. Gao, M. Guzzi, J. Huston, P. Nadolsky, J. Pumplin, C. Schmidt, D. Stump and C. P. Yuan, New parton distribution functions from a global analysis of quantum 
chromodynamics, Phys. Rev. D93 (2016), no. 3, 033006, arXiv:1506.07443 [hep-ph]].

[37] L. A. Harland-Lang, A. D. Martin, P. Motylinski and R. S. Thorne, Parton distributions in the LHC era: MMHT 2014 PDFs, Eur. Phys. J. C75 (2015), no. 5, 204, arXiv:1412.3989 hep$\mathrm{ph}]$.

[38] E. Bothmann, M. Schönherr and S. Schumann, Reweighting QCD matrix-element and partonshower calculations, arXiv: 1606.08753 [hep-ph]. 